\title{
Dependence of convective flows and particle precipitation in the high-latitude dayside ionosphere on theXandYcomponents of the interplanetary magnetic field
}

Article

Published Version

Cowley, S. W. H., Morelli, J. P. and Lockwood, M. (1991) Dependence of convective flows and particle precipitation in the high-latitude dayside ionosphere on theXandYcomponents of the interplanetary magnetic field. Journal of Geophysical Research, 96 (A4). p. 5557. ISSN 0148-0227 doi: https://doi.org/10.1029/90JA02063 Available at https://centaur.reading.ac.uk/38847/

It is advisable to refer to the publisher's version if you intend to cite from the work. See Guidance on citing.

Published version at: http://dx.doi.org/10.1029/90JA02063

To link to this article DOI: http://dx.doi.org/10.1029/90JA02063

Publisher: American Geophysical Union

All outputs in CentAUR are protected by Intellectual Property Rights law, including copyright law. Copyright and IPR is retained by the creators or other copyright holders. Terms and conditions for use of this material are defined in the End User Agreement. 


\section{www.reading.ac.uk/centaur}

\section{CentAUR}

Central Archive at the University of Reading

Reading's research outputs online 


\title{
Dependence of Convective Flows and Particle Precipitation in the High-Latitude Dayside Ionosphere on the $X$ and $Y$ Components of the Interplanetary Magnetic Field
}

\author{
S. W. H. COWLEY AND J. P. MORELLI \\ Blackett Laboratory, Imperial College, London \\ M. LOCKWOOD ${ }^{1}$ \\ Rutherford-Appleton Laboratory, Chilton, Didcot, Oxon
}

\begin{abstract}
The asymmetries in the convective flows, current systems, and particle precipitation in the high-latitude dayside ionosphere which are related to the equatorial plane components of the interplanetary magnetic field (IMF) are discussed in relation to the results of several recent observational sudies. It is argued that all of the effects reported to date which are ascribed to the y component of the IMF can be understood, at least qualitatively, in terms of a simple theoretical picture in which the effects result from the stresses exerted on the magnetosphere consequent on the interconnection of terrestrial and interplanetary fields. In particular, relaxation under the action of these stresses allows, in effect, a partial penetration of the IMF into the magnetospheric cavity, such that the sense of the expected asymmetry effects on closed field lines can be understood, to zeroth order, in terms of the "dipole plus uniform field" model. In particular, in response to IMF By, the dayside cusp should be displaced in longitude about noon in the same sense as $B y$ in the northem hemisphere, and in the opposite sense to $B y$ in the southem hemisphere, while simultaneously the auroral oval as a whole should be shifted in the dawn-dusk direction in the opposite sense with respect to $B y$. These expected displacements are found to be consistent with recently published observations. Similar considerations lead to the suggestion that the auroral oval may also undergo displacements in the noon-midnight direction which are associated with the $x$ component of the IMF. We show that a previously published study of the position of the auroral oval contains strong initial evidence for the existence of this effect. However, recent results on variations in the latitude of the cusp are more ambiguous. This topic therefore requires further study before definitive conclusions can be drawn.
\end{abstract}

\section{INTRODUCTION}

The structure and dynamics of the Earth's magnetosphere are principally determined by the large-scale convection of plasma and magnetic flux within the cavity, which is driven by momentum transfer from the solar wind. The magnitude and form of this flow is known to depend significantly on the strength and direction of the interplanetary magnetic field (IMF), thus constituting one of the major indirect pieces of evidence which indicates that magnetic reconnection at the magnetopause plays a substantial role in exciting the flow (see, for example, the reviews by Cowley [1983, 1986], and references therein). In particular, the north-south (z) component of the IMF is a key parameter determining the amount of open flux in the magnetosphere and the overall magnitude of the internal flow, as measured, for example, by the size of the polar cap at ionospheric heights and the total potential drop across it, respectively [Holzworth and Meng, 1975, 1984; Reiff et al., 1981; Doyle and Burke, 1983; Wygant et al., 1983; Cowley, 1984; Holzer et al., 1986]. However, the east-west (y) component of the IMF also plays an important role and is known to be associated with a number of dawn-dusk magnetospheric flow and plasma asymmetries which reverse in sense between the northern and southern hemispheres, and with the polarity of By [see Cowley, 1981a, and references therein; Cowley and Hughes, 1983; Burch et $a l ., 1985$ ]. In addition, it has also been suggested on theoretical grounds that further asymmetries may occur which are explicitly associated with the sunward-tailward $(x)$ component of the IMF [Cowley, 1981b]. However, observational evidence for the expected effects in this case is marginal at present. In this report

Copyright 1991 by the American Geophysical Union.

Paper number 90JA02063.

0148-0227/91/90JA-02063\$05.00 we will be concerned with the latter two groups of effects, associated with the $x$ and $y$ components of the IMF.

In a recent paper, Newell et al. [1989] have presented a valuable and detailed study of the IMF dependence of various properties of the dayside cusp region, as identified in ion and electron data obtained on a large number of passes by the low-altitude $(838 \mathrm{~km})$ polar-orbiting DMSP F-7 (Defense Meteorological Satellite Program) spacecraft. The "cusp" refers to that region where magnetosheath plasma precipitates essentially unmodified down to the ionosphere and is morphologically distinct from the "cleft" where the precipitation is of characteristically lower density and higher energy, and is taken to originate in the low-latitude boundary layer. One of the principal results of this study was to show that the center of the cusp precipitation region shifts in local time in response to the $B_{y}$ component of the IMF, thus substantiating earlier indications to this effect presented by Candidi et al. [1983]. The results obtained by Newell et al. [1989] imply that in the northern hemisphere the cusp is displaced toward dusk when $B_{y}$ is positive, and toward dawn when $B_{y}$ is negative, with opposite displacements in the southem hemisphere. This effect was also shown to be larger when $B_{z}$ is negative than when it is positive. Newell et al. [1989] also investigated the possibility of a latitudinal shift in the position of the cusp associated with the $B_{x}$ component of the IMF, as suggested by the theoretical work cited above, and reported negative results. Here we wish to comment briefly on both these findings and their bearing on related results published recently by Holzworth and Meng [1984], Erlandson et al. [1988], Lu et al. [1989], and Candidi et al.[ 1989].

\section{EFFECTS ASSOCIATED WITH THE $Y$ COMPONENT OF THE IMF}

We first consider the effects on dayside ionospheric flows and precipitation patterns which are thought to be associated with the 
$B_{y}$ component of the IMF. Our prime objective is to discuss the apparent discrepancy, noted by Newell et al. [1989], between the $B_{y}$-dependent dawn-dusk displacements of the cusp outlined above, and the related displacements of the auroral oval as a whole found by Holzworth and Meng [1984] (and by many other authors referred to below). We will also point out the relationship between these results and those of three other recent studies, by Candidi et al. [1989] on IMF $B_{y}$-dependent local time variations of the latitude of the cusp, by $L u$ et al. [1989] on the By-dependence of some features of the dayside flow pattem, and by Erlandson et al. [1988] on the $B_{y}$-dependence of dayside field-aligned current (FAC) systems.

The first topic to be considered here is thus the relationship between the results on the local time of the dayside cusp precipitation zone determined by Newell et al. [1989], and the earlier findings on the auroral oval by Holzworth and Meng [1984]. The latter authors fitted circles to DMSP images of the auroral oval, and investigated possible shifts in the position of the center of the circle with IMF $B_{y}$. Results showing how the auroral oval moves in the dawn-dusk direction in response to $B_{y}$ are shown here in Figure 1, taken from Table 1 of Holzworth and Meng [1984]. We have adopted the results derived from examination of images of the sunward and tailward portions of the auroral oval (the "Sun-tail" data), for which the determination of the dawn-dusk position of the center of the auroral circle should be most accurate, and which then have been combined with hourly averaged IMF data from the same hour as the auroral observations (the " $\Delta T=0$ " results). Figure 1 shows that for $B_{y}$ positive the auroral oval is

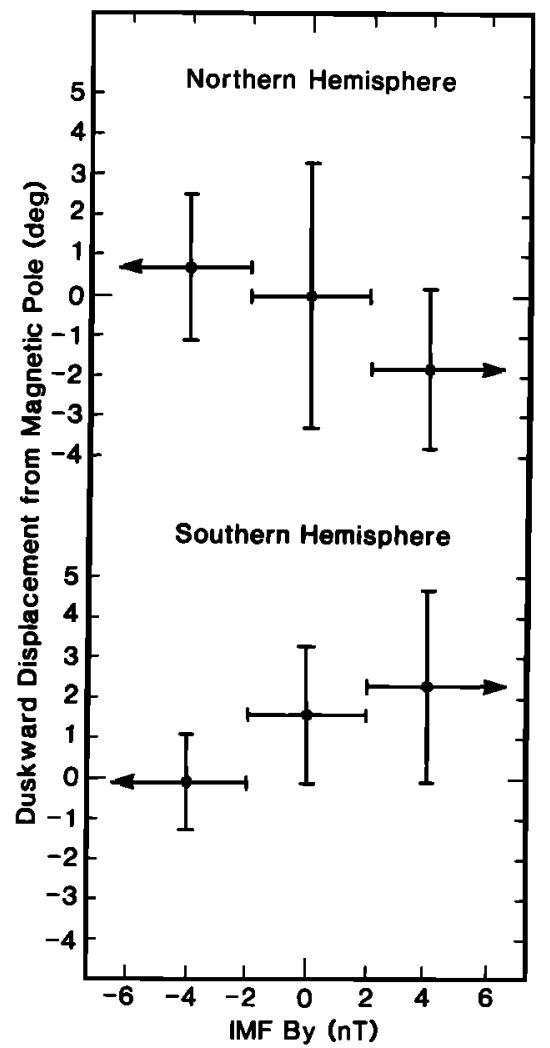

Fig. 1. Dependence of the dawn-dusk position of the center of the auroral oval on IMF $B_{y}$, as determined from DMSP images by Holzworth and Meng [1984] and taken from their Table 1. The dats subset determined from images of the sunward and tailward sectors of the auroral oval (the "Sun-tail" data) is shown and is combined with hourly averaged IMF values determined over the same hour as the auroral images (the " $\Delta T=0$ " data). shifted toward dawn in the northem hemisphere and toward dusk in the southem hemisphere, and vice versa for $B_{y}$ negative. The overall displacement of the oval between periods of positive and negative $B_{y}\left(B_{y} \sim \pm 4 \mathrm{nT}\right)$ is typically $\sim 2.5^{\circ}$ in each hemisphere. It should be pointed out that not all the data subsets in Holzworth and Meng's study show such a clear effect in the northerm hemisphere as that depicted in Figure 1. However, many previous and subsequent studies, mainly concemed with measurements of ionospheric flow (specifically the latitude of the sunward-antisunward flow reversals near the dawn-dusk meridian), confirm the reality of the effect [Heppner, 1972; Mozer et al., 1974; $Z i$ et al., 1987; Heppner and Maynard, 1987; Hairston and Heelis, 1990]. Here we are assuming, of course, that the auroral oval and the ionospheric flow pattem vary together in such a way as to maintain a consistent mutual relationship, as seems physically reasonable, and as indicated by observation [Heelis et al., 1980].

It is pointed out by Newell et al. [1989], however, that the displacements of the cusp found in their study have the opposite sense with respect to IMF $B_{y}$ to the displacements of the oval derived by Holzworth and Meng [1984]. As mentioned above, for $B_{y}$ positive, Newell et al. [1989] found that the cusp is shifted duskward in the northem hemisphere, and dawnward in the southern hemisphere, and vice versa for $B_{y}$ negative, opposite to the sense of the shift of the auroral oval as a whole shown in Figure 1. Newell et al. [1989] conjectured that the fits to the auroral oval could be influenced by nightside effects which might respond differently to IMF $\boldsymbol{B}_{\boldsymbol{y}}$.

The simultaneous appearance of these oppositely directed displacements can, however, be understood in basic terms within the framework of the "dipole plus uniform field" picture [Dungey, 1963; Cowley, 1973; Stern, 1973; Lyons, 1985], as sketched in Figure 2. This figure may be taken to represent a view of the Earth's field from the direction of the Sun, and illustrates the distortions in the dipole field lines (dashed lines) which occur when a uniform field, pointing in the positive $y$ direction (left to right), is added. This field will have the same sense as the $B_{y}$ field

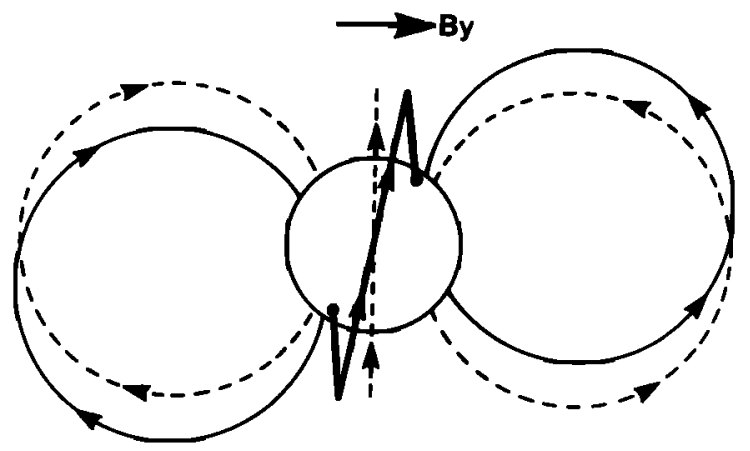

Fig. 2. Sketch showing the perturbations of the Earth's dipole field lines (dashed lines) produced by adding a uniform field directed from left to right. Field lines lying initially in a meridian plane transverse to the added field are tilted out of the meridian as shown at the center of the diagram, such that their footprints at the Earth are displaced in longitude in the same direction as the added field in the northem hemisphere, and in the opposite direction to the added field in the sonthem hemisphere. Simultaneously, field lines lying in the meridian containing the direction of the added field, as shown on the left and right of the diagram, have their footprints shifted in latitude, in the opposite direction to the added field in the northem hemisphere, and in the same direction as the added field in the southem hemisphere. The field line footprints therefore undergo oppositely directed displacements in the meridian containing the added field and in the plane transverse to the latter. 
in the interplanetary medium and arises from the stresses exerted on the magnetosphere which result from the interconnection of the terrestrial and interplanetary fields, as will be discussed further below. It is evident from the figure that field lines lying initially in the noon-midnight meridian plane will be tilted by the added $B_{y}$ field such that their footprints at the Earth will be displaced in local time in the direction of the added field in the northern hemisphere, and in the opposite direction to the added field in the southern hemisphere. These longitudinal displacements are then consistent in direction with the local time shifts of the dayside cusp determined by Newell et al. [1989], as noted by the latter authors. However, it is also clear that these longitudinal shifts do not reflect the expected displacement of the auroral oval as a whole. Rather, as can be seen from the figure, field lines lying in the dawn-dusk meridian will simultaneously be displaced in latitude, in the opposite direction to the added field in the northern hemisphere, and in the same direction as the added field in the southern hemisphere. These shifts are then consistent with the dawn-dusk displacements of the auroral oval as a whole found by Holzworth and Meng [1984] (and other authors), as displayed in Figure 1.

We will now indicate briefly why the highly simplistic "dipole plus uniform field" picture is germane in these considerations. The effects discussed here result fundamentally from the electromagnetic stresses which are exerted upon the magnetosphere as a result of the interconnection which occurs between the magnetospheric and solar wind magnetic fields. In the presence of IMF $B_{y}$, for example, oppositely directed east-west stresses are exerted on open flux tubes in the northern and southern hemispheres, these stresses forming a torque on the magnetosphere which is directed sunward for $B_{y}$ positive, and antisunward for $B_{y}$ negative. These stresses result in an asymmetrical evolution of the open flux tubes over the dayside magnetopause, and an asymmetrical transport into the tail, such that, for example, for IMF $B_{y}$ positive, open tubes are preferentially transported to the dawnside of the tail lobe in the northern hemisphere, and to the duskside of the tail lobe in the southern hemisphere, and vice versa for IMF $B_{y}$ negative. This asymmetrical transport is directly related to the $B_{y}$-dependent east-west flows on newly opened flux tubes in the dayside cusp ionosphere which give rise to the Svalgaard-Mansurov effect, as will be discussed further below. However, as a result of this transport, the open tubes in the tail lobes become tilted in the direction of the IMF $B_{y}$ field, as pointed out previously by Cowley [1981a], and as demonstrated observationally by Fairfield [1979] and Tsurutani et al. [1984]. That is to say, there results, in effect, a partial penetration of the IMF $B_{y}$ field into the open field line region of the magnetosphere. However, the asymmetric evolution of the open flux tubes from the dayside into the tail will also result in stresses being communicated to the interior region of closed field lines as well. For example, when $B_{y}$ is positive, the asymmetrical transport of open tubes into the tail will lead to asymmetrical inward compressive forces being exterted on closed flux tubes in the interior, which will be strongest on the dawnside in the northern hemisphere and on the duskside in the southern hemisphere (and vice versa for $B_{y}$ negative). These forces will cause the interior closed field lines to become distorted in a sense which (for IMF $B_{y}$ positive) will be qualitatively similar to those depicted here in Figure 2. These distortions of closed field lines will thus be associated with a perturbation magnetic field which has the same sense as the interplanetary $B_{y}$ field, such that in effect the IMF $B_{y}$ field will penetrate the region of closed field lines as well. The latter effect has been confirmed by Cowley and Hughes [1983] using observations at the geostationary orbit, and by Crooker et al. [1987] from observations in the region just inside the dayside magnetopause. The general principles which are involved here may be simply summarized. As a result of dayside reconnection, the terrestrial and interplanetary fields become interconnected. Electromagnetic stresses are consequently exerted on the magnetospheric open flux tubes, the direction of the stresses being govemed by the orientation of the IMF. In response, the system may be expected to relax under the action of these stresses toward a state of reduced electromagnetic stress. The limiting case of zero electromagnetic stress in the presence of an interplanetary field and reconnection is then the "dipole plus uniform field" paradigm, toward which we may thus expect the magnetosphere to relax, at least partially.

The consequences of the above discussion for the properties of flows in the dayside ionosphere in the northem hemisphere are summarized in Figure 3, where the top panel shows flows for IMF $B_{y}$ positive, while the bottom panel shows flows for IMF $B_{y}$ negative. In these sketches the solid lines show the plasma streamlines, the dashed line is the boundary between open and closed field lines, and the circled dots and crosses show the upward- and downward-directed FACs, respectively, which are principally associated with shears in the flow. Specifically, the currents shown in the equatorward part of the flow pattem are the "region 2" currents, those located at the flow shear near the open-closed field line boundary are the "region 1 " currents, while the currents within the region of open field lines are the "cusp" currents.

In drawing these sketches three main asymmetry effects have been represented. First, the boundary between open and closed field lines is shown displaced toward dawn for positive $B_{y}$ and toward dusk for negative $B_{y}$, in conformity with the auroral observations of Holzworth and Meng [1984], and with the flow

(a) IMF By>0

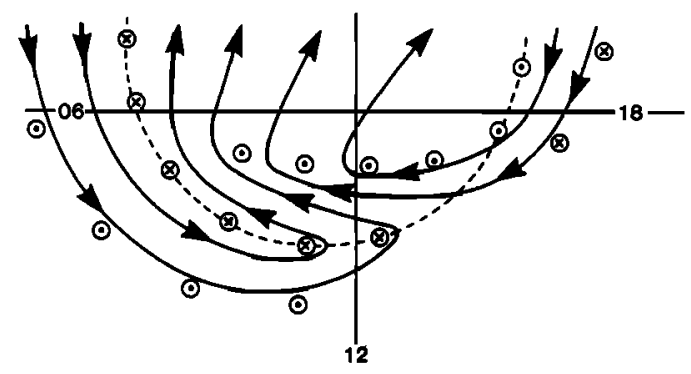

(b) IMF By<0

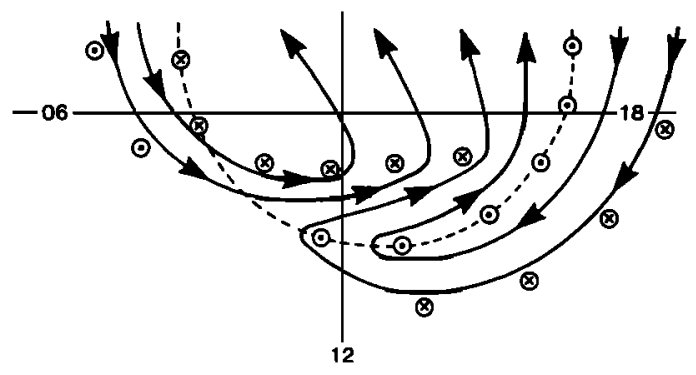

Fig. 3. Sketch showing the MMF $B_{y}$-dependent effects on the streamlines (solid curves) and on the open-closed field line boundary (dashed curve) in the northem hemisphere. The circled symbols represent the FAC flow, circled dots represent upward current, and circled crosses downward current. Top Conditions for IMF $B_{y}$ positive and bottom negative. The "zero potential" streamline referred to in section 2 (not shown explicitly), is the streamline which separates the dawn and dusk convection cells, and lies between the inner pair of streamlines drawn in each of the sketches. 
observations of Heppner [1972], Mozer et al. [1974], Zi et al. [1987], Heppner and Maynard [1987], and Hairston and Heelis [1990]. The oppositely directed shifts present simultaneously in the southern hemisphere clearly require the closed field lines in and near the dawn-dusk meridian to undergo IMF $B_{y}$-dependent latitudinal displacements of the character described by the "dipole plus uniform field" model, as illustrated in Figure 2.

Second, we have shown the center of the flow across the open-closed field line boundary to be shifted in local time about noon, to dusk for $B_{y}$ positive, and to dawn for $B_{y}$ negative. This displacement is consistent with the shifts in the local time of the equatorward boundary of the cusp precipitation found by Newell et al. [1989], using the physically plausible assumption that the cusp is formed where the streamlines cross the open-closed field line boundary, and in the region immediately poleward thereof (with the highest energy particles arriving just poleward of the boundary, and lower energy particles further downstream due to their longer time-of-flight from the magnetopause). The streamlines in the empirical flow models described by Heppner and Maynard [1987] show a corresponding shift in the same sense, though the effect is not symmetrical about noon. The displacement of the center of the flow at the open-closed boundary near noon is also compatible with the $B_{y}$-dependence of the "region 1" currents discovered in Viking data by Erlandson et al. [1988], who found a local time displacement about noon of the reversal in the polarity of the "region 1" current which is consistent with that shown in Figure 3. These authors also showed that the equatorward boundary of the region of intense magnetosheath precipitation lay within the "region 1" current, but poleward of the latter's equatorward boundary, a result which is also consistent with the above discussion. Again, the oppositely directed local time displacements which must simultaneously be present in the southem hemisphere require closed field lines in and near the noon meridian to tilt in the manner described by the "dipole plus uniform field" model, leading to asymmetric longitudinal shifts of the field line footpoints.

It should be noted that an immediate consequence of the configuration shown in Figure 3 is that the cusp/cleft precipitation in the dayside oval, mapping to the vicinity of the open-closed field line boundary, should show an IMF $B_{y}$-dependent difference in latitude between the prenoon and postnoon sectors. As a consequence of the dawn-dusk shift of the boundary, the cusp/cleft should be located at higher latitudes postnoon than prenoon in the northern hemisphere for $B_{y}$ positive, and vice versa for $B_{y}$ negative. In the southem hemisphere the latitudinal displacements should be in the opposite direction. Using near-simultaneous overpasses of the cusp/cleft region at different local times by two DMSP spacecraft, Candidi et al. [1989] have recently presented evidence indicating the existence of these displacements. We may point out, however, that the basic effect is already inherent in the dawn-dusk displacements of the auroral oval found in the optical studies of Holzworth and Meng [1984].

The third principal effect shown in Figure 3 concems the direction of the flow on open field lines in the region immediately poleward of the open-closed field line boundary. In the northem hemisphere these flows are predominantly westward for $B_{y}$ positive and eastward for $B_{y}$ negative, in conformity with the Svalgaard-Mansurov effect mentioned above, before tuming to move across the central polar region at higher latitudes. In general, the motion of the open field lines is expected to respond both to the east-west-directed stresses which result from their connection to the IMF in the presence of $B_{y}$, as discussed above, and also to the antisolar-directed stresses which result from the flow of the magnetosheath plasma. Initially, the $B_{y}$-dependent stresses are expected to be dominant on newly opened flux tubes, giving rise to the east-west flows just poleward of the open-closed field line boundary, as outlined above, and as described originally by Atkinson [1972] and Jørgensen et al. [1972]. However, as the open tubes relax, and as the magnetosheath flow speed increases away from the subsolar region, control transfers from the magnetosheath field to the magnetosheath flow, and the open tubes correspondingly turn to move over the central polar cap, as described previously by Saunders [1989]. The point at which the transfer of control takes place is marked in the ionosphere by the position of the cusp FAC. As can be seen from Figure 3, in the northern hemisphere these currents are expected to be predominantly upward directed and centered prenoon for IMF By positive, and to be predominantly downward directed and centered postnoon for $B_{y}$ negative, in conformity with the observations presented by Iijima et al. [1978], McDiarmid et al., [1978], and Erlandson et al. [1988].

With the aid of Figure 3 we can now finally discuss the recent results on the $B_{y}$-dependence of the dayside ionospheric flow presented by $L u$ et al. [1989]. Using flow observations from a large number of orbits of the AE-C. AE-D, and DE-2 spacecraft, these authors examined the electrostatic potential between the plasmapause at low latitudes and the "polar cap" flow reversal and the way in which this potential varies with local time under different IMF conditions. In particular, they determined how the "zero potential" line, the streamline separating the dawn and dusk convection cells, moves in local time in response to IMF $B_{y}$. They found that in the northem hemisphere this line shifts toward dawn for IMF $B_{y}$ positive, and toward dusk for $B_{y}$ negative, and vice versa in the southern hemisphere. These displacements are thus again opposite to those determined for the dayside cusp precipitation by Newell et al. [1989].

With reference to Figure 3 it is easily seen, however, that the "zero potential" streamline shifts in different directions in different parts of the dayside flow. In the vicinity of the open-closed field line boundary and "region 1" current, for example, it is shifted toward dusk in the northem hemisphere for $B_{y}$ positive, and toward dawn for $B_{y}$ negative, while further poleward, in the vicinity of the cusp current the shifts are opposite in direction. It is made clear in Lu et al.'s [1989] discussion that in the noon sector it was the latter boundary, colocated with the cusp current, which was taken to correspond to the "polar cap" boundary, and not the former boundary, colocated with the "region 1" current. Consequently, the oppositely directed shifts found in Lu et al.'s [1989] study and that of Newell et al. [1989] are readily understood in terms of Figure 3. If the "region 1" boundary had been chosen, then as noted by $L u$ et al. [1989], the shifts derived would be expected to reverse in sense, corresponding to the shifts reported by Newell et al. [1989] with regard to the cusp precipitation, and by Erlandson et al. [1988] with regard to the FAC polarity reversal, as mentioned above.

In summary, it seems reasonable to conclude that all of the IMF $B_{y}$-related asymmetry effects reported in the literature to date can be understood, at least qualitatively, within a simple theoretical framework. The effects result fundamentally from the stresses exerted on the magnetosphere due to the interconnection between the terrestrial and interplanetary magnetic fields, leading to dawn-dusk asymmetries in the presence of IMF $B_{y}$ on both open and closed flux tubes. One result of the action of the stresses is to cause, in effect, a partial penetration of the IMF $B_{y}$ field into the magnetospheric cavity, and the sense of the asymmetry effects involving closed flux tubes (specifically the displacements of the 
cusp and auroral oval) can consequently be understood in terms of the simplistic "dipole plus uniform field" model.

\section{EFFECTS ASSOCIATED WITH THE $X$ COMPONENT OF THE IMF}

The general considerations outlined above conceming the significance of the "dipole plus uniform field" model lead naturally to the expectation that magnetospheric asymmetry effects might also occur which are associated explicitly with $B_{x_{0}}$ as discussed previously by Cowley [1981b]. In this case the "dipole plus uniform field" picture indicates that field lines in the dawn-dusk meridian will undergo longitudinal shifts which are expected to lead to little observational effect, while field lines in the noon-midnight meridian will be shifted in latitude, leading to sunward-tailward shifts of the polar cap and auroral oval as a whole (see Figure 2). Specifically, for $\boldsymbol{B}_{\boldsymbol{x}}$ positive the auroral oval and polar cap should be shifted tailward in the northern hemisphere and sunward in the southern hemisphere, and vice versa for $B_{\mathbf{x}}$ negative.

Newell et al. [1989] comment that no such effects were found in Holzworth and Meng's [1984] study of DMSP images. However, we would question whether this statement is fully justified. In fact, Holzworth and Meng [1984] reported no separate study of the effects of $B_{x}$ alone but concentrated principally on divisions of the data according to IMF $B_{y}$, such as those previously displayed in Figure 1. (They did report the results of a study of possible sunward-tailward shifts predicted by Gonzales and Mozer [1974] associated with the product $B_{x} B_{\mathrm{z}}$, with a negative outcome, but that of course is quite another matter.) However, it is well known that $B_{z}$ and $B_{y}$ are strongly anticorrelated in the solar wind, as a consequence of the average Parker spiral direction in the interplanetary magnetic field. Consequently, if an IMF $B_{x}$ effect is indeed present in Holzworth and Meng's [1984] auroral data, it would be expected to appear in their study as an IMF $B_{y}$-dependent sunward-tailward shift of the auroral oval, such that for positive $B_{y}$ (corresponding to negative $B_{z}$ ) the northern hemisphere oval is displaced sunward and the southem hemisphere oval tailward, and vice versa for negative $B_{y}$ (corresponding to positive $B_{x}$ ). Such an effect is indeed present in the data, though only the southem hemisphere displacement was remarked upon by Holzworth and Meng [1984]. The results are shown in Figure 4 in the same format as Figure 1, again taken from Holzworth and Meng's [1984] Table 1. A clear effect of the appropriate sign is apparent in both hemispheres, with a total displacement of $\sim 2.5^{\circ}$ between positive and negative $B_{y}$ conditions. The magnitude of the effect is therefore directly comparable with the dawn-dusk displacements shown previously in Figure 1. We thus argue that Holzworth and Meng's [1984] results contain strong prima facie evidence for the existence of the expected IMF $B_{x}$ effect. Of course, we cannot be sure from the results derived that the sunward-tailward displacements found by Holzworth and Meng [1984] are indeed associated with IMF $B_{x}$ rather than with some unexpected phenomenon actually associated with IMF $B_{y}$, though it seems physically plausible to suggest that the former is the case rather than the latter. In this context we would point out that many of the effects ascribed to $B_{y}$ would also show a very strong correlation with IMF $B_{x}$ as well, due to the spiral geometry of the interplanetary field, and are only ascribed specifically to $B_{y}$ on grounds of physical plausibility. Similarly, we would ascribe the sunward-tailward displacements of the auroral oval discovered by Holzworth and Meng [1984] to a $B_{x}$ effect on the same basis. Clearly, a much more careful analysis of a much larger quantity of data would be required to unambiguously determine which effects are associated explicitly with $B_{\boldsymbol{x}}$, and which with $B_{\mathbf{y}}$.

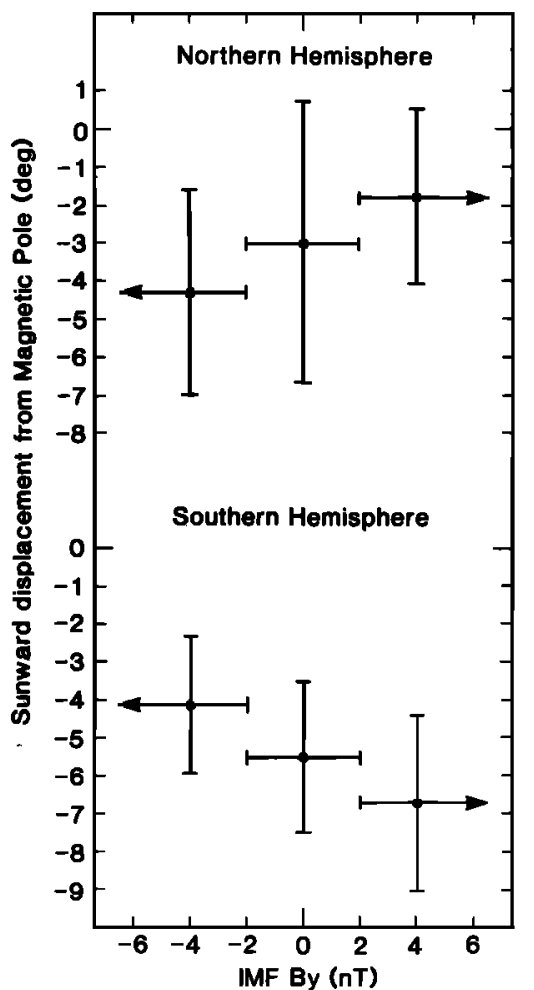

Fig. 4. Dependence of the sunward-tailward position of the center of the auroral oval on IMF $B_{y}$, as determined from DMSP images by Holzworth and Meng [1984] and taken from their Table 1 . The format is the same as in Figure 1 . Here we ascribe the underlying physical effect to IMF $B_{x_{n}}$ with which MF $B$ is strongly anticorrelated in the solar wind.

We finally wish to comment on the results obtained by Newell et al. [1989] on the IMF $B_{x}$ dependence of the latitude of the cusp, as determined from their study of DMSP particle data. For purposes of comparison we first show in Figure 5 the "expected" IMF-dependence of the boundary between the polar cap and the dayside auroral oval in the northern hemisphere, as determined using the "dipole plus uniform field" model. Specifically, we plot the latitude at the Earth's surface in the northern hemisphere of the boundary between open and closed field lines in the model, determined by numerical integration, versus magnetic local time (MLT) between 0600 and 1800 hours on the dayside. In each case we have taken the uniform "interplanetary field" to have a strength of $7.5 \mathrm{nT}$, and to be directed with a polar angle lying $30^{\circ}$ below the dipole equatorial plane. We have therefore taken a constant IMF $B_{2}=-3.75 \mathrm{nT}$ along the dipole axis, while the IMF component in the plane of the dipole equator has a constant strength of $B_{e} \simeq 6.50 \mathrm{nT}$, but various azimuthal orientations. The dipole field is such that its strength at the equator at the Earth's surface is $3 \times 10^{4} \mathrm{nT}$.

In the top panel of Figure 5 we show the effect on the boundary latitude associated with the IMF $B_{x}$ component alone, i.e., we have taken the equatorial IMF to point either directly away from the Sun (solid curve, corresponding to IMF $B_{x}$ negative), or directly toward the Sun (dashed curve, corresponding to IMF $B_{x}$ positive). It can be seen that in this case the variation of the boundary latinde is symmetrical about noon and corresponds to a tailward displacement of the northern polar cap for $B_{x}$ positive, and a sunward displacement for $B_{x}$ negative, in conformity with the discussion given above. The peak displacement of the boundary between the two $B_{x}$ polarities thus occurs at noon in this case, where the amplitude is $\sim 2.5^{\circ}$ as found in the observational study 


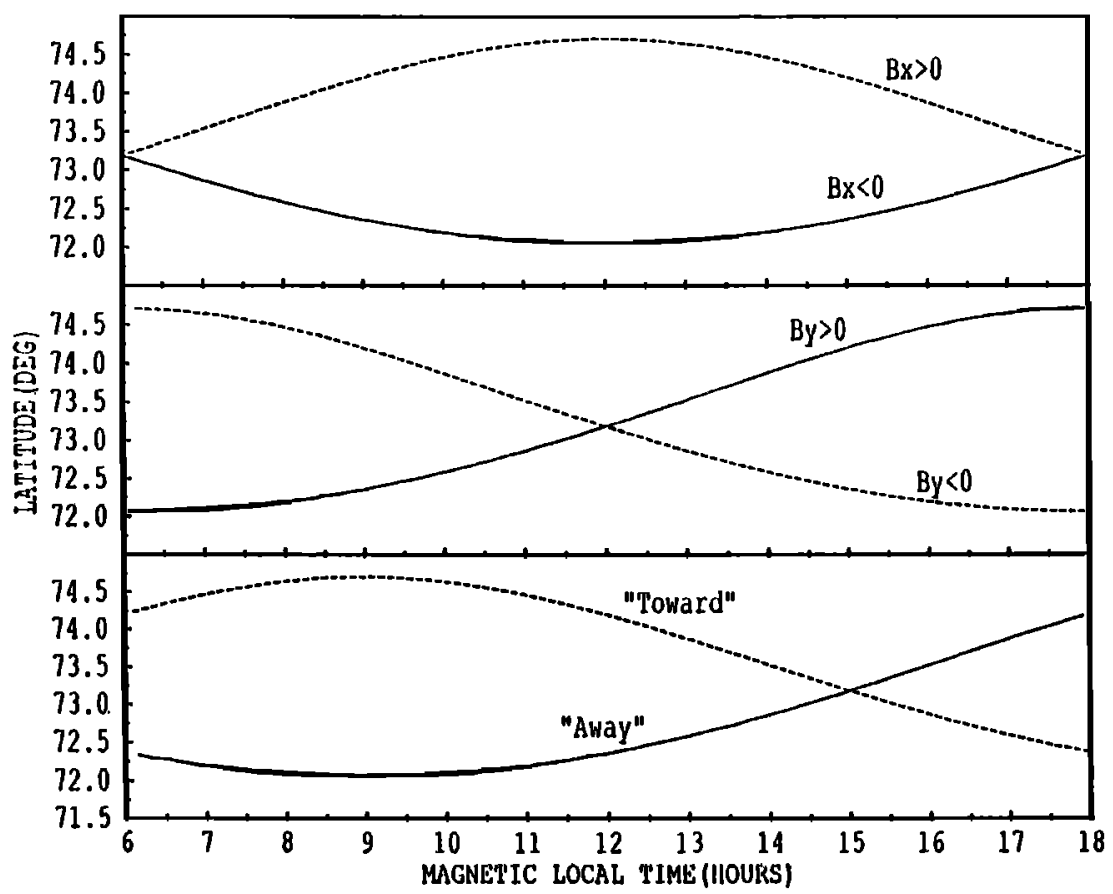

Fig. 5. Plots of the latitude of the boundary between open and closed field lines versus MLT, in the "dipole plus uniform field" model, for various orientations of the added uniform field. The latter field has a total strength of $7.5 \mathrm{nT}$, with a constant component along the dipole moment vector of $B_{2}=-3.75 \mathrm{nT}$. The added field in the equatorial plane of the dipole thus has a strength of $=$ $6.50 \mathrm{nT}$, with various orientations as indicated in the panels of the figure. The dipole field is such that at the Earth's surface the equatorial field has a strength of $3 \times 10^{4} \mathrm{nT}$. Top Boundary when the equatorial field lies in the negative (solid curve) and positive (dashed curve) $x$ directions. Similarly, (middle) corresponding results for equatorial fields in the positive (solid curve) and negative (dashed curve) $y$ direction. Finally, (bottom) results for equatorial fields which lie along the Parker spiral direction at $45^{\circ}$ to the Earth-Sun line, where the solid curve corresponds to an "away" field $\left(B_{x}<0, B_{y}>0\right)$, and the dashed curve to a "toward" field $\left(B_{x}>0\right.$, $B_{y}<0$ ).

by Holzworth and Meng [1984], and goes to zero at the dawn-dusk meridian.

Similarly, the middle panel of Figure 5 shows the effect associated with the IMF $B_{y}$ component alone, i.e. we have taken the equatorial component of the IMF to point either directly in the positive $y$ direction (solid curve), or in the negative $y$ direction (dashed curve). In this case the variation of the boundary latitude with local time corresponds to a dawnward shift of the boundary for $B_{y}$ positive, and a duskward shift for $B_{y}$ negative, again in agreement with the previous discussion. The peak displacements thus now occur on the dawn-dusk meridian, and there is no latitudinal variation at noon. However, the $B_{y}$-dependent gradient in the latitude of the boundary at noon corresponds to the local time effect found in the latitude of the cusp/cleft precipitation by Candidi et al. [1989], as was mentioned above.

Finally, the bottom panel of Figure 5 corresponds to the "average" case where the IMF lies along the Parker spiral direction, at $45^{\circ}$ to the Earth-Sun line. The solid curve corresponds to an "away" sector $\left(B_{x}<0, B_{y}>0\right)$, while the dashed curve corresponds to a "toward" sector $\left(B_{x}>0, B_{y}<0\right)$. In this case the displacements are maximum at $0900 \mathrm{MLT}$ and go to zero at 1500 MLT.

Overall, the numerical results shown in Figure 5 can be summarized by saying that the polar cap boundary in the northern hemisphere becomes displaced in a direction which is opposite to that of the added field in the equatorial plane of the dipole, as previously stated. Thus as the IMF changes its polarity, the maximum displacement of the auroral oval and polar cap occurs at the local time corresponding to the azimuth of the added field, while no displacement occurs at the local time corresponding to the directions which are orthogonal to added field. Thus a pure $B_{x}$ effect will show a maximum displacement at noon and no response at dawn and dusk, and vice versa for a pure $B_{y}$ effect. On the other hand, if both components of the IMF are equally effective in causing displacements of the polar cap, then we would expect to find maximum displacements near to $0900 \mathrm{MLT}$, and no response near to $1500 \mathrm{MLT}$, due to the average spiral direction of the IMF.

With these expectations in mind, we now tum to the results on the cusp latitude determined by Newell et al. [1989], and shown here in Figure 6 (reproduced from Figure $4 b$ of Newell et al. [1989]). This shows the average latitude of the equatorward boundary of the northern hemisphere cusp plotted versus MLT, for data with IMF $B_{2}$ negative (for which the effects should be maximal), and split according to IMF $B_{x}>3 \mathrm{nT}$ (dashed curve), and IMF $B_{x}<-3 \mathrm{nT}$ (solid curve). It should be pointed out, however, that due to the average spiral geometry of the IMF these splits of the data will also be strongly biased in IMF $B_{y}$ as well, with $B_{y}<0$ for the dashed curve and $B_{y}>0$ for the solid curve, such that both $B_{x}$ and $B_{y}$ effects will simultaneously be present, if indeed both occur. We may thus initially anticipate that the results should be comparable with the bottom panel of Figure 5 for "toward" and "away" fields. (Note that solid and dashed curves have comparable meanings in the two figures). However, it can be seen that the displacements observed in the prenoon sector, between 1000-1100 MLT, are actually opposite to those anticipated (and those found by Holzworth and Meng [1984]), with the cusp being displaced sunward by $\sim 1^{\circ}$ when $B_{x}$ is positive compared with when it is negative. In the absence of published error estimates, however, it is unclear whether these displacements are statistically significant, particularly given the relatively low probability of 


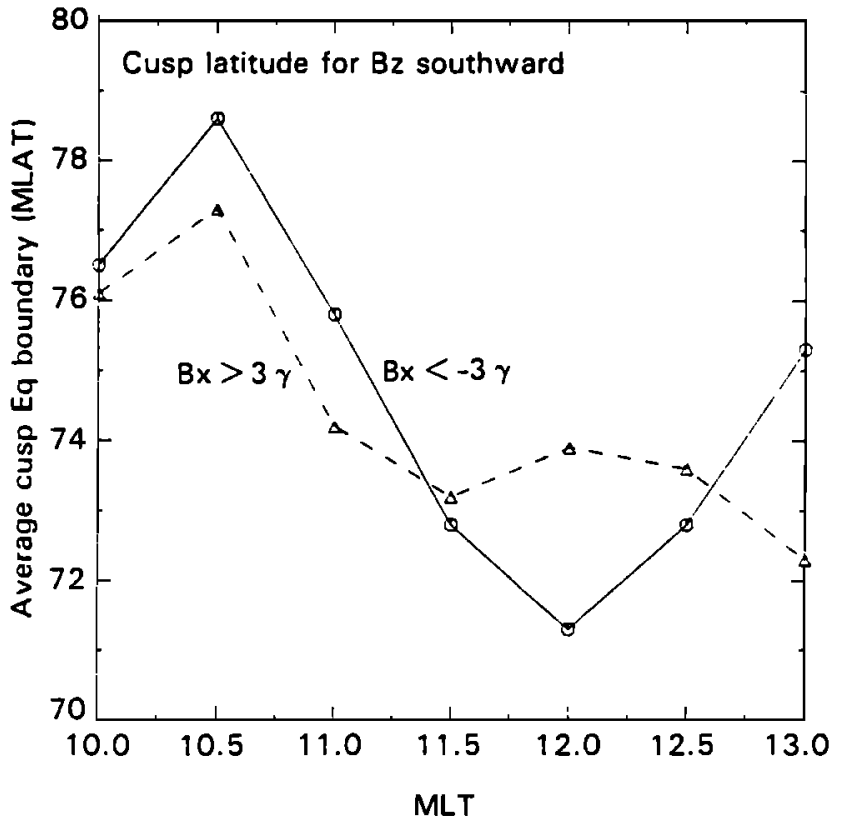

Fig. 6. Average latitude versus MLT of the equatorward boundary of the cusp precipitation region in the northem hemisphere, for IMF $B_{2}<0$ and IMF $B_{x}>3$ nT (dashed curve), and IMF $B_{x}<-3$ nT (solid curve), taken from Newell et al. [1989, Figure $4 b$.] The dashed and solid curves have similar meanings to those in Figure 5.

observing the cusp in these MLT intervals ( $~ 20 \%$ probablility for $B_{y}>3 \mathrm{nT}$ and $B_{z}<0$, and $\sim 50 \%$ probability for $B_{y}<-3 \mathrm{nT}$ and $B_{z}<0$, as indicated in Figures $3 b$ and $3 d$ of Newell et al. [1989]).

In the noon sector in Figure 6, where the probability of observing the cusp is high $(\sim 80 \%)$ for both signs of the IMF, an oppositely directed latitudinal displacement of the cusp of $\sim 2.5^{\circ}$ is observed, consistent with the expected $B_{x}$ effect. However, the displacement again reverses in sense at 1300 MLT. Taken at face value (and ignoring the prenoon results), this latter behavior might be taken as evidence of a predominant $B_{y}$ effect in the data as in the middle panel of Figure 5 (though the magnitude of the observed effect is much larger than that in the middle panel of Figure 5 close to noon), with a weak $B_{x}$ effect present as well, associated with the displacement of the cross-over point into the early postnoon sector. We note in this context that the geosynchronous perturbation fields are also rather less convincing in the $x$ component than in the $y$ component, particularly for IMF $B_{z}$ negative [Hughes and Cowley, 1986]. However, such a conclusion does not appear to be consistent with the results obtained by Holzworth and Meng [1984], who found dawn-dusk and noon-midnight shifts of comparable magnitude, which would instead lead us to expect a result similar to that shown in the bottom panel of Figure 5, with significant latitudinal shifts throughout the local time zone covered by Figure 6 .

The results in Figure 6 are also somewhat unexpected in that the mean absolute latitude of the equatorward boundary of the cusp shows large variations with local time. Previous analyses of the cusp boundaries determined from the DMSP F7 data, though undifferentiated with respect to the IMF, do not show such an effect. Rather, within the local time interval shown in Figure 6, the overall mean equatorward boundary of the cusp was found to lie at a relatively constant latitude between $\sim 75^{\circ}$ and $76^{\circ}$ (see Figure 5 of Newell and Meng [1988]). We might then have expected Figure 6 also to show a relatively constant mean latitude, but one which is somewhat lower due to the selection for IMF $\boldsymbol{B}_{\mathbf{z}}$ negative. Instead, the mean boundary in Figure 6 is located at $-77^{\circ}$ prior to $11 \mathrm{MLT}$, which is distinctly unexpected, and at $\sim 73^{\circ}$ at later local times. The significance of these results remains unclear but may point toward some peculiarity in the data distribution which was used to construct Figure 6. In particular, it might be profitable to examine whether there are any significant differences in the mean negative IMF $B_{z}$ fields which are associated with the individual data points in Figure 6 , since the latitude of the cusp is rather more strongly dependent on IMF $B_{2}$ than on the equatorial plane components of the IMF (see Figure 5 of Newell et al. [1989]). Overall, however, it seems clear that this topic is one which requires further careful study to establish whether or not the expected effect actually occurs. We would suggest, however, that in view of the results presented by Holzworth and Meng [1984], it is somewhat premature to conclude that $B_{x}$ effects do not, in fact, occur in the magnetosphere. Other $B_{x}$ effects predicted by Cowley [1981b] and yet to be investigated in any detail include north-south asymmetries in the properties of the plasma mantle in the tail lobes, and north-south displacements of the central tail current sheet.

\section{SUMMARY}

In this paper we have discussed the patterns of convective flow and particle precipitation which occur in the high-latitude dayside ionosphere, and their dependence on the $x$ and $y$ components of the IMF. Our particular purpose has been to point out the relationship between the observational results recently presented on this topic by Holzworth and Meng [1984], Erlandson et al. [1988], Newell et al. [1989], Lu et al. [1989] and Candidi et al. [1989], and the results of other authors. The main points we wish to emphasize are as follows.

1. The existence of asymmetry effects at high latitudes associated with the equatorial components of the IMF results from the stresses exerted upon the magnetosphere consequent upon its magnetic interconnection with the interplanetary magnetic field. Relaxation under the action of these stresses results, in effect, in a partial penetration of the interplanetary field inside the cavity, on both open and closed field lines. The asymmetry effects occurring on closed field lines can thus be described in terms of a distortion of the magnetic field which to zeroth order can be modelled by the "dipole plus uniform field" picture. Thus in the presence of IMF $B_{y}$ the cusp will be displaced in local time away from noon in the same direction as $B_{y}$ in the northern hemisphere, and in the opposite direction to $B_{y}$ in the southem hemisphere. Simultaneously, the auroral oval and polar cap as a whole will be shifted latitudinally in the other direction, opposite to $B_{y}$ in the northern hemisphere, and in the same direction as $B_{y}$ in the southem hemisphere. These expectations have been shown to be qualitatively consistent with Newell et al.'s [1989] results on the position of the cusp, Erlandson et al.'s [1988] results on dayside FAC systems, Holzworth and Meng's [1984] findings on the position of the auroral oval as a whole (and the corresponding flow results of Heppner [1972], Mozer et al. [1974], Zi et al. [1987], Heppner and Maynard [1987], and Hairston and Heelis [1990]), and Candidi et al.'s [1989] results on variations of the cusp/cleft latitude with local time. In the open field line region within the polar cap the flow is mainly east-west in the region just poleward of the open-closed field line boundary, as a direct result of the stresses exerted on the flux whes by their comnection to the IMF. These flows are directed opposite to $B_{y}$ in the northem hemisphere, and in the same direction as $B_{y}$ in the southern hemisphere, consistent with the Svalgaard-Mansurov effect. Further poleward. the antisunward flow over the polar cap is, in the northem hemisphere, correspondingly concentrated on the dawnside for $B_{y}$ positive, and on the duskside for $B_{y}$ negative, and vice versa in the 
southern hemisphere. These effects are responsible for the $B_{y}$-dependent displacements of the "zero potential" streamline found recently by $L u$ et $a l$. [1989], and for the $B_{y}$-dependence of the cusp FAC found by lijima et al. [1978], McDiarmid et al. [1978], and Erlandson et al. [1988].

2. Simple theory also indicates that related effects should occur in association with IMF $B_{\boldsymbol{x}}$ as well, the principal effect at high latitudes being a noon-midnight shift of the open-closed field line boundary and auroral oval, in the opposite direction to $B_{x}$ in the northern hemisphere, and in the same direction as $B_{x}$ in the southern hemisphere. The results presented by Holzworth and Meng [1984] contain significant evidence indicating the reality of the effect (though not noted by them), while Newell et al.'s [1989] cusp study is not clear in this regard. Further investigation of this effect is thus required. However, we regard it as premature to conclude that asymmetry effects associated with IMF $B_{x}$ do not occur within the magnetosphere.

Ackowledgment. This work was performed while one of us (J.P.M.) was supported by a SERC postgraduate studentship.

\section{REFERENCES}

Atkinson, G., Magnetospheric flows and substorms, in Magnetosphere-IonosphereInteractions, edited by K. Folkestadt, p. 203, Universitetsforlaget, Oslo, Norway, 1972.

Burch, J. L., P. H. Reiff, J. D. Menietti, R. A. Heelis, W. B. Hanson, S. D. Shawhan, E. G. Shelly, M. Sugiura, D. R. Weimer, and J. D. Winningham, IMF By-dependent plasma flow and Birkeland currents in the dayside magnelosphere, 1, Dynamics Explorer observations, $J$. Geophys. Res., 90, 1577, 1985.

Candidi, M., H. W. Kroehl, and C.-I. Meng, Intensity distribution of dayside polar soft electron precipitation and the IMF, Planet. Space Sci., $31,489,1983$.

Candidi, M., G. Mastrantonio, S. Orsini, and C.-I. Meng, Evidence for the influence of the interplanetary magnetic field azimuthal component on polar cusp configuration, J. Geophys. Res., 94, 13585, 1989.

Cowley, S. W. H., A qualitative study of the reconnection between the Earth's magnetic field and an interplanetary magnetic field of arbitrary orientation, Radio Sci., 8, 903, 1973.

Cowley, S. W. H., Magnetospheric asymmetries associated with the Y-component of the IMF, Planet. Space Sci, 29, 79, 1981 a.

Cowley, S. W. H., Asymmetry effects associated with the $X$-component of the IMF in a magnetically open magnetosphere, Planet. Space Sci., 29. $809,1981 b$.

Cowley, S. W. H., Interpretation of observed relations between solar wind characteristics and effects at ionospheric altitudes, in High Latitude Space Plasma Physics, edited by B. Hultqvist and T. Hagfors, p. 225, Plenum, New York, 1983.

Cowley, S. W. H., Solar wind control of magnetospheric convection, in Achievements of the IMS, ESA SP-217, p. 483, ESTEC, Noordwijk, The Netherlands, 1984.

Cowley, S. W. H., The impact of recent observations on theoretical understanding of solar wind-magnetosphere interactions, J. Geomagn. Geoelectr., 38, 1223, 1986.

Cowley, S. W. H., and W. J. Hughes, Observation of an IMF effect in the $Y$ magnetic field component at geostationary orbit, Planet. Space Sci., 31 , 73, 1983.

Crooker, N. U., J. Berchem, and C. T. Russell, Cusp displacement at the magnetopause for large IMF Y component, J. Geophys. Res., 92, 13467, 1987.

Doyle, M. A., and W. J. Burke, S 3-2 measurements of the polar cap potential, J. Geophys. Res., 88, 9125, 1983.

Dungey, J. W., The structure of the exosphere or adventures in velocity space, in Geophysics, The Earth's Environment, edited by C. DeWitt, J. Hieblot, and L. LeBeav, p. 503, Gordon and Breach, New York, 1963.

Erlandson, R. E., L. J. Zanetti, T. A. Potemra, P. F. Bythrow, and R. Lundin, IMF $B_{y}$ dependence of region 1 Birkeland currents near noon, $J$. Geophys. Res., 93, 9804, 1988.

Fairfield, D. H., On the average configuration of the geomagnetic tail, $J$. Geophys. Res., 84, 1950, 1979.

Gonzales, W. D., and F. S. Mozer, A quantitative model for the potential resulting from reconnection with an arbitrary interplanetary magnetic field, J. Geophys. Res., 79, 4186, 1974.

Hairston, M. R., and R. A. Heelis, Model of the high-latitude ionospheric convection partem during southward interplanetary magnetic field using DE 2 data, J. Geophys. Res., 95, 2333, 1990.

Heelis, R. A., J. D. Winningham, W. B. Hanson, and J. L.Burch, The relationships between high-latitude convection reversals and the energetic particle morphology observed by Amospheric Explorer, $J$. Geophys. Res., 85, 3315, 1980.

Heppner, J. P., Polar cap electric field distributions related to the interplanetary magnetic field direction, J. Geophys. Res., 77, 4877, 1972.

Heppner, J. P., and N. C. Maynard, Empirical high-latitude electric field models, J. Geophys. Res., 92, 4467, 1987.

Holzer, R. E., R. L. McPherron, and D. A. Hardy, A quantitative empirical model of the magnetospheric flux transfer process, J. Geophys. Res., 91, 3287, 1986.

Holzworth, R. H., and C.-I. Meng. Mathematical representation of the auroral oval, Geophys. Res. Lett., 2, 377, 1975.

Holzworth, R. H., and C.-I. Meng, Auroral boundary variations and the interplanetary magnetic field, Planet. Space Sci., 32, 25, 1984.

Hughes, W. J., and S. W. H. Cowley, Observation of MM-associated magnetic field perturbations in the GSM $X$ component at geostationary orbit, in Solar-Wind Magnetosphere Coupling, edited by Y. Kamide and J. A. Slavin, p. 691, Terra Science, Tokyo, 1986.

Iijima, T., R. Fujii, T. A. Potemra, and N. A. Saflekos, Field-aligned currents in the south polar cusp and their relationship to the interplanetary magnetic field, J. Geophys. Res., 83, 5595, 1978.

Jørgensen, T. S., E. Friis-Christensen, and J. Wilhjelm, Interplanetary magnetic field direction and high latitude ionospheric currents, $J$. Geophys. Res., 77, 1976, 1972.

Lu, G., P. H. Reiff, M. R. Hairston, R. A. Heelis, and J. L. Karty, Distribution of convection potential around the polar cap boundary as a function of the interplanetary magnetic field, J. Geophys. Res., 94, $13447,1989$.

Lyons, L. R., A simple model for polar cap convection pattems and generation of $\theta$ auroras, J. Geophys. Res., 90, 1561, 1985.

McDiarmid, I. B., J. R. Burrows, and M. D. Wilson, Magnetic field perturbations in the dayside cleft and their relationship to the IMF, $J$. Geophys. Res., 83, 5753, 1978.

Mozer, F. S., W. D. Gonzales, F. Bogott, M. C. Kelley, and S. Schutz, High-latitude electric fields and the three-dimensional interaction between the interplanetary and terrestrial magnetic fields, $J$. Geophys. Res., 79, 56, 1974.

Newell, P. T., and C.I. Meng, The cusp and cleft/boundary layer: Low-altitude identification and statistical local time variation, $J$. Geophys. Res., 93, 14549, 1988.

Newell, P. T., C.-I Meng, D. G. Sibeck, and R. Lepping, Some low-altitude cusp dependencies on the interplanetary magnetic field, $J$. Geophys. Res., 94, 8921, 1989.

Reiff, P. H., R. W. Spiro, and T. W. Hill, Dependence of polar cap potential drop on interplanetary parameters, J. Geophys. Res., 86, 7639, 1981.

Saunders, M. A., Origin of the cusp Birkeland currents, Geophys. Res. Lett., 16, 151, 1989.

Stem, D. P., A study of the electric field in an open magnetospheric model, J. Geophys. Res., 78, 7292, 1973.

Tsurutani, B. T., D. E. Jones, R. P. Lepping, E. J. Smith, and D. G. Sibeck, The relationship between the IMF $B_{y}$ and the distant tail (150-238 $\left.R_{E}\right)$ lobe and plasma sheet $B_{y}$ fields, Geophys. Res. Lett., 11, 1082, 1984.

Wygant, J. R., R. B. Torber, and F. S. Mozer, Comparison of S 3-3 polar cap potential drops with the interplanetary magnetic field and models of magnetopause reconnection, J. Geophys. Res., 88, 5727, 1983.

Zi, M.-Y., E. Nielsen, W. B. Hanson, and T. A. Potemra, Polar convection and Birkeland currents during strongly positive IMF $B_{\mathbf{y}}, J$. Geophys. Res., 92, 3417, 1987.

S. W. H. Cowley and J. P. Morelli, Blacketu Laboratory, Imperial College, London SW7 2BZ, England.

M. Lockwood, Rutherford-Appleton Laboratory, Chilton, Didcot, Oxon OX11 OQX, England.

(Received June 7, 1990; revised September 3, 1990; accepted September 3, 1990.) 